\title{
Tumor-derived vascular endothelial growth factor (VEGF)-A facilitates tumor metastasis through the VEGF-VEGFR1 signaling pathway
}

\author{
WENBO LIU ${ }^{1}$, JIYING XU ${ }^{2}$, MINGMING WANG ${ }^{2}$, QIANGXIU WANG ${ }^{3}$, YULI BI $^{2}$ and MINGYONG HAN ${ }^{2}$ \\ ${ }^{1}$ Department of Immunology, Shandong Qianfoshan Hospital, Shandong University, 16766 Jingshi \\ Road, Jinan 250014; ${ }^{2}$ Cancer Therapy and Research Center; ${ }^{3}$ Department of Pathology, Shandong \\ Provincial Hospital, Shandong University, 324 Jingwuweiqi Road, Jinan 250021, P.R. China
}

Received May 12, 2011; Accepted June 23, 2011

DOI: 10.3892/ijo.2011.1138

\begin{abstract}
Vascular endothelial growth factor (VEGF) and its receptors are involved in carcinogenesis, invasion and tumor angiogenesis, but the underlying mechanism by which VEGF promotes tumor metastasis is poorly understood. In this study, we show that in cancer patients high expression of VEGF is correlated with metastasis, and anti-VEGF treatment (bevacizumab) has clinical effects on tumor metastasis. Two human lung carcinoma cell lines (A549 and SPCA1 cells) with distinct VEGF expression were injected intravenously through the lateral tail vein of SCID mice and a murine model was developed. We investigated the association between the expression of VEGF and tumor metastasis by microvessel density, immunohistochemistry and whole mount staining. At sacrifice, in the high VEGF expression A549 cell line group, the induced tumor was distinctively larger in size and multiple metastatic lesions were found in lung tissues. Two specific neutralizing anti-mouse VEGFR1 and VEGFR2 antibodies were administered to the tumor-bearing mice; anti-VEGFR1, but not anti-VEGFR2 treatment produced inhibitive effects on VEGFinduced tumor metastasis. These findings demonstrate that the VEGF-VEGFR1 signaling pathway is crucial for tumor metastasis and the blockade of VEGF-VEGFR1-induced metastasis may provide a novel approach for the prevention and treatment of tumor metastasis.
\end{abstract}

\section{Introduction}

Vascular endothelial growth factor (VEGF)-A, usually referred to as VEGF, is one of the most potent and studied angiogenic factors $(1,2)$. VEGF exerts its angiogenic effects by interacting

Correspondence to: Dr Mingyong Han, Cancer Therapy Center, Shandong Provincial Hospital, Shandong University, 324 Jingwuweiqi Road, 250021 Jinan, P.R. China

E-mail: hanmingyong71@126.com

Key words: vascular endothelial growth factor, metastasis, signal pathway, VEGFR with specific receptors on endothelial cells stimulating their growth and differentiating into blood vessels $(3,4)$. VEGF is a key regulator of physiological angiogenesis during embryogenesis, skeletal growth and reproductive functions (5). VEGF has been implicated in pathological angiogenesis associated with tumors, intraocular neovascular disorders and other conditions (6). VEGF is also known as vascular permeability factor, based on its ability to induce vascular leakage. Consistent with a role in the regulation of vascular permeability, VEGF induces endothelial fenestration in some vascular beds $(7,8)$. The biological effects of VEGF are mediated by two receptor tyrosine kinases (RTKs), VEGFR1 and VEGFR2, which differ considerably in signaling properties. VEGFR1 mediates the signal pathway which plays important roles in pathological conditions such as cancer, ischemia and inflammation, whereas VEGFR2 mediates most of the endothelial growth and survival signals.

Metastasis, the spread of malignant cells from a primary tumor to distant sites, poses the biggest problem to cancer treatment and is the main cause of death of cancer patients (9). Tumor progression towards metastasis is a multistage process. In this process, tumor cell invasion alone is not sufficient to produce distant metastasis, it requires the transport of malignant cells through blood and/or lymph vessels, so tumor angiogenesis is one of the hallmarks of cancer. Malignant tumors stimulate blood vessel growth by producing angiogenic factors, and some angiogenic factors contribute to tumor cells metastasis (10-14). Among the tumor-derived growth factors, VEGF is a key angiogenic factor most frequently used by tumors and other tissues to switch on their angiogenic phenotypes (15-18).

In solid tumors, VEGF and its receptors are involved in carcinogenesis, invasion and tumor angiogenesis. High expression levels of VEGF in tumors have been correlated with enhanced tumor growth $(15,19,20)$, but there are few studies aiming at the correlation between tumor VEGF expression and metastasis. The underlying mechanism of angiogenesis factors and tumor metastasis is poorly understood. The objectives of this study are to elucidate the effects of VEGF expression in tumor metastasis in cancer patients and in a mouse xenograft tumor model. 


\section{Materials and methods}

Patients. A total of 109 patients with lung cancer were enrolled between June 2007 and November 2009. None of the patients had received prior chemotherapy or radiotherapy, 71 patients had advanced stage non-small cell lung cancer (NSCLC) and one experienced recurrence after lobotomy. Inclusion criteria for the patients were: i) cytologically or histologically proven lung cancer; and ii) clinical and pathological stages of tumors were established according to TNM stage classification system 2002. Tissue biopsy was performed during surgical operations of patients. The study was performed after approval by the Human Investigations Committee of our institute. Written informed consent was obtained from each patient prior to the start of the study.

Treatment protocols. Thirty-eight patients were enrolled in the trial to evaluate the efficacy of bevacizumab. Patients were randomly assigned on a 1:1 basis to bevacizumab (Avastin; Genetech, South San Francisco, CA) in combination with chemotherapy or chemotherapy alone. Chemotherapy regimen was docetaxel + DDP (docetaxel, Sanofi-Aventis, Bridgewater, NJ; DDP, Qilu company, Jinan, China). Patients who experienced toxicity or disease progression as determined by the investigator were discontinued from the treatment of the study. The dose of bevacizumab in this study was $15 \mathrm{mg} / \mathrm{kg}$ administered by intravenous infusion on the first day of each 3-week cycle. For chemotherapy, patients were treated with infusion of $75 \mathrm{mg} / \mathrm{m}^{2}$ of DDP and $75 \mathrm{mg} / \mathrm{m}^{2}$ of docetaxel on the first day of cycle $(1$ cycle $=21$ days). Premeditation regimens for docetaxel and DDP were administered as described in the respective prescribing information for each drug. Patients received full supportive care as needed, including hematopoietic growth factors, and antibiotics.

Tumor evaluation. Patients underwent tumor assessments at baseline and after two cycles of therapy. Tumor response was prospectively assessed according to RECIST criteria by computerized tomography-scan. Toxicity was assessed according to the Common Toxicity Criteria version 2.0 (21).

Samples and tissue specimens. Peripheral blood samples were obtained from the patients before surgery and chemotherapy. For serum collection, blood samples were drawn into a vacationer containing sodium ethylenediaminetetraacetic acid and immediately centrifuged at $3,000 \mathrm{rpm}$. Serum samples were collected and stored at $-20^{\circ} \mathrm{C}$ until VEGF measurements were taken. Tumor tissues were collected from patients operated for NSCLC at the Shandong Provincial Hospital, Jinan, China from 2005 to 2009. Suitable formalin fixed, paraffin-embedded material was available.

Cells and animals. Cells were cultured in RPMI-1640 with $10 \%$ fetal calf serum (FCS) medium. Proliferation assays were performed using the MTT assay (Roche; Palo Alto, CA, USA) following the manufacturer's protocol. Immunoincompetent 6-8-week-old female SCID mice were acclimated and caged in groups of 6 or less. Animals were followed up to 3-6 weeks and anesthetized by an injection of a mixture of hypnorm (fentanyl citrate, fluanisone; VetaPharma) and midazolam (dormicum;
Roche) (1:1) before all procedures and sacrificed by a lethal dose of $\mathrm{CO}_{2}$, followed by cervical dislocation. All animal studies were reviewed and approved by the animal care and use committee of the Shandong Provincal Animal Board (Jinan, China).

Reagents. The antibodies used in our studies included a rat anti-mouse CD31 monoclonal antibody (BD-Pharmingen), a goat anti-rat Alex-555 red (Invitrogen), a rat anti-mouse VEGFR1 (MF1) antibody and a rat anti-mouse VEGFR2 (DC101) antibody (ImClone Systems Inc.)

Tumor cell proliferation assay. A 72-h tumor cell proliferation assay was performed. Cells growing in gelatinized 6-well plates were dispersed in $0.25 \%$ tyrosine solution and resuspended with DMEM containing 0.5\% FCS. We added $1 \times 10^{4}$ cells to each gelatinized well of 24-well plates and incubated them at $37^{\circ} \mathrm{C}$ for $1 \mathrm{~h}$. After a total of $72 \mathrm{~h}$ of incubation, cells were trypsinized, resuspended in Isoton II solution (Beckman Coulter), and counted with a Coulter counter (Beckman Coulter) (22).

Tumor growth assay. SPCA1 cells and A549 cells $\left(1 \times 10^{6}\right)$, suspended in $0.1 \mathrm{ml}$ of PBS, were subcutaneously injected into the flanks of SCID mice. Tumor growth was measured every 3 days, and tumor volume was calculated as following: tumor volume $=$ length $\times(\text { width })^{2}(23)$.

Metastasis assay. Tumor cells were labeled with DiI, then tumor cells $\left(1 \times 10^{5}\right)$ were resuspended in $0.1 \mathrm{ml}$ PBS and injected intravenously through the lateral tail vein of the SCID mice, two weeks after injection, animals were sacrificed and lungs were resected. Surface metastasis were examined visually under a light microscopy, further scanned for $\mathrm{DiI}$-postive metastasis, and processed for histological analysis. The total number of metastatic foci per reference area was quantified (24).

Histology process and immunohistochemical analysis. Malignant and non-malignant paraffin-embedded tissues were sectioned in $5 \mu \mathrm{m}$ thickness and stained with $\mathrm{H} \& \mathrm{E}$ according to our previously described methods. A representative tissue block of the resected lung cancer tissue, which contained tumor, was selected from each case for immunohistochemical studies. Formalin-fixed, paraffin-embedded $4-\mu \mathrm{m}$ thick tumor tissue sections were dried for $1 \mathrm{~h}$ at $60^{\circ} \mathrm{C}$, de-paraffinized in tissue clear and dehydrated in a graded ethanol series, and treated with $3 \%$ hydrogen peroxide to block endogenous peroxidase activity. Antigen retrieval was performed by immersing slides in TEG pH 9.0 Tris EDTA pH 8.0 for VEGF, VEGFR1 and VEGFR2 and microwaving at high power for $15 \mathrm{~min}$. Non-immune serum was used to block non-specific binding. Afterwards the sections were incubated with primary monoclonal antibodies: VEGF (Lab Vision, clone JH121) at a dilution of 1:50 for 60 min, VEGFR1 and VEGFR2 (RB-9239-P from Lab Vision) at a dilution of 1:50 for $30 \mathrm{~min}$. The antibody was visualized using the Dako EnVision + system and diaminobenzidine as a chromogen in an automated immunostainer (DakoCytomation, TechMate $^{\mathrm{TM}}$ Horizon, Glostrup, Denmark). The sections were reinforced by $\mathrm{CuSO}_{4}$ and counterstained with haematoxylin. Negative controls had the antibody replaced by phosphatebuffered saline. 
Table I. Patient characteristics and serum VEGF levels.

\begin{tabular}{lccc}
\hline Characteristics & Number & $\begin{array}{c}\text { VEGF level } \\
(\mathrm{pg} / \mathrm{ml})\end{array}$ & P-value \\
\hline Gender & 72 & $79.3 \pm 23.6$ & \\
$\quad$ Male & 37 & $84.5 \pm 34.4$ & 0.247 \\
Female & & & \\
Age & 64 & $92.7 \pm 48.3$ & \\
$\quad$ 60 & 45 & $86.4 \pm 27.3$ & 0.327 \\
$>60$ & & & \\
Histology & 68 & $77.9 \pm 21.3$ & \\
Adenocarcinoma & 41 & $81.7 \pm 49.0$ & 0.374 \\
Squamous carcinoma & & & \\
Pleural effusion & 96 & $62.9 \pm 34.6$ & \\
Negative & 13 & $134.8 \pm 29.6$ & 0.007 \\
Positive & & & \\
Stage & 38 & $66.4 \pm 34.7$ & \\
$\quad$ I + II & 71 & $127.7 \pm 46.2$ & 0.018 \\
III + IV & & & \\
\hline
\end{tabular}

Whole-mount staining. Whole mount staining was performed according to published methods. Briefly, small pieces of tissues were cut into thin slices and fixed in 3\% PFA overnight, followed by treatment with proteinase $\mathrm{K}(20 \mu \mathrm{g} / \mathrm{ml})$. Rat anti-mouse CD31 antibodies were used as primary antibodies and goat anti-rat Alex-555 red (Invitrogen) antibodies were used as secondary antibodies. Slides were examined under a confocal microscope (Zeiss Confocal LSM510 microscope). By scanning 10 thin sections (4-5 $\mu \mathrm{m}$ distances) of each sample, 3 dimensional images of each tissue sample were assembled. Quantitative analysis from at least 5 different tissue sections was performed using the color range tool of Adobe Photoshop CS software program.

Measurement of VEGF in serum or culuture medium. VEGF levels in serum or culture medium samples were assessed using the Quantikine ELISA kits (R\&D Systems Inc.). The assay employs a quantitative sandwich enzyme according to manufacturer's instruction and the standardized protocol. The minimum detectable concentration of VEGF was $1.6 \mathrm{pg} / \mathrm{ml}$. The method was prepared to recognize both VEGF-121 and VEGF-165, which represented the most biologically active molecular forms of total circulating VEGF.

Statistical analyses. The statistical analyses were done using JMP version 6 . The groups were compared using t-test and Fischer's exact test. The analyses yielded $95 \%$ confidence intervals and $\mathrm{P}$-values. Values of $\mathrm{P}<0.05$ were considered statistically significant.

\section{Results}

Patient characteristics and serum VEGF level. The patient characteristics are listed in Table I. Of the 109 patients, 72 were male $(66.1 \%), 37$ were female $(33.9 \%)$, and the median age was 53.7 years (range, 43-72). Sixty-eight patients $(51.4 \%)$ had adenocarcinoma and 13 patients (11.9\%) had pleural effusion. According to WHO TNM stage, there were 38 patients in I and II stages and 71 patients in III and IV stages. For serum VEGF level, there was no difference between the patients, sex, histological type, but the difference between pleural effusionpositive group and negative group was significant $(\mathrm{P}<0.01)$. The difference between disease stage I and II group and III and IV group was also significant $(\mathrm{P}<0.05)$.

Correlation between serum VEGF level and metastasis in cancer patients. To further analyze the correlation between serum VEGF level and metastasis in cancer patients, we found that the circulating VEGF level in cancer patients without metastasis was $66.8 \pm 16.7 \mathrm{ng} / \mathrm{ml}$, in cancer patients with only lymphatic metastasis was $74.3 \pm 26.4 \mathrm{ng} / \mathrm{ml}$ and in cancer patients with organ metastasis was $98.8 \pm 23.4 \mathrm{ng} / \mathrm{ml}$. There is no difference between the group of patients without metastasis and the group with lymphatic metastasis, but the difference between non-metastasis group and organ metastasis group is significant $(\mathrm{P}<0.01)$ (Fig. 1).
A

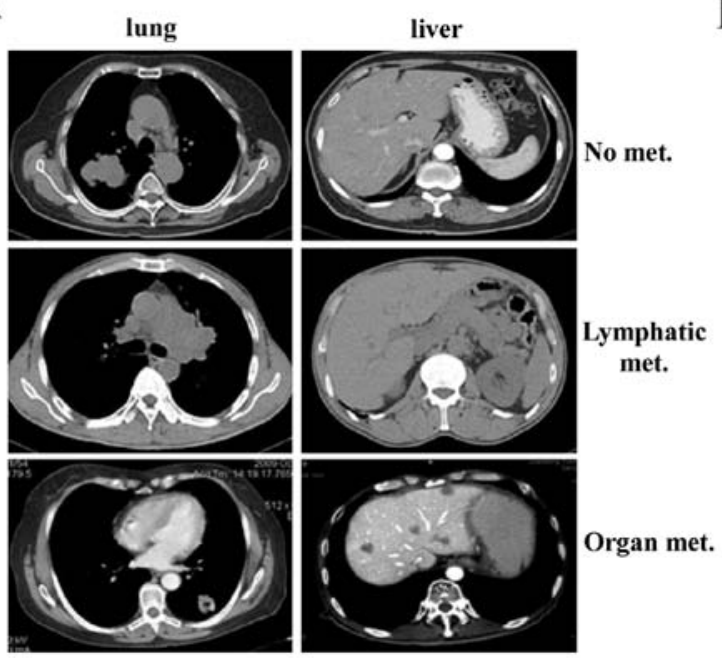

B

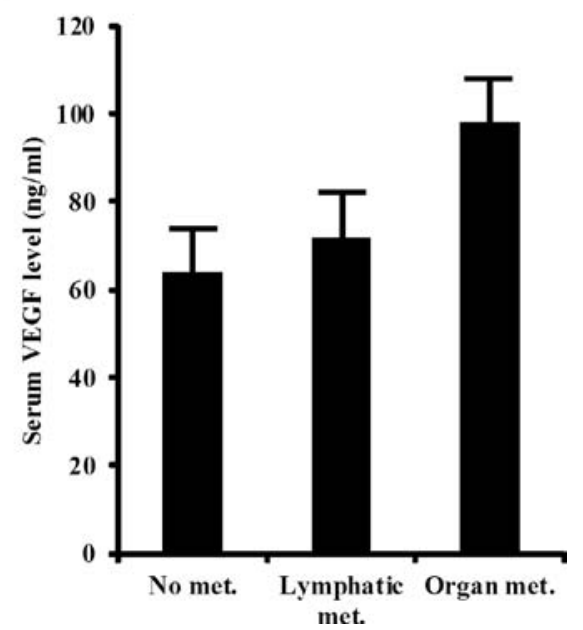

Figure 1. The relationship of tumor metastasis and serum VEGF level in lung cancer patients. 
Table II. Distribution of VEGF expression as measured by percentage divided in metastasis.

\begin{tabular}{lccccrr}
\hline & No cells stained & $<10 \%$ cells stained & $10-25 \%$ cells stained & $25-75 \%$ cells stained & $>75 \%$ cells stained & Total \\
\hline NM $(\%)$ & $0(0.0)$ & $3(12.5)$ & $9(28.0)$ & $11(45.8)$ & $1(4.2)$ & 24 \\
LM $(\%)$ & $1(2.4)$ & $5(12.2)$ & $14(34.1)$ & $16(39.0)$ & $6(14.6)$ & 41 \\
OM $(\%)$ & $0(0.0)$ & $2(4.5)$ & $8(18.2)$ & $19(43.2)$ & $14(31.8)$ & 44 \\
Total $(\%)$ & $1(0.9)$ & $10(9.2)$ & $31(28.4)$ & $46(42.2)$ & $21(19.3)$ & 109 \\
\hline
\end{tabular}

NM, no metastasis; LM, lymphatic metastasis; OM, organ metastasis.

Table III. Response to treatment with chemotherapy alone or bevacizumab and chemotherapy.

\begin{tabular}{lcccc}
\hline & $\mathrm{CR}$ & $\mathrm{PR}$ & $\mathrm{SD}$ & $\mathrm{PD}$ \\
\hline $\mathrm{C}(\%)$ & $0(0.0)$ & $4(21.1)$ & $8(42.1)$ & $7(36.8)$ \\
$\mathrm{B}+\mathrm{C}(\%)$ & $1(5.3)$ & $6(31.6)$ & $7(36.8)$ & $5(26.3)$ \\
\hline
\end{tabular}

B, bevacizumab; C, chemotherapy.

Correlation between VEGF expressions in tumor tissue and tumor metastasis in cancer patients. VEGF expressions in tumor tissues varied from absent to intermediate and strong. Positive staining was obtained in 108 out of 109 cases (99.1\%), and typical immunohistochemical staining is shown in Fig. 2A. VEGF immunoreactivity was observed mainly in the cytoplasm of tumor cells, and also frequently in stromal cells. The distribution of VEGF-staining was not continuous in the whole slide of the specimen. Regarding the propotion of positively stained malignant cells in each tumor, a significant difference was observed between non-metastasis group having lower percent of positive tumor cells and to the group with organs metastasis. $(\mathrm{P}=0.002)$. There was no statistically significant correlation between non-metastasis group and lymphatic metastasis group (Table II).

Effects of anti-VEGF treatment on tumor metastasis. A total of 38 patients were randomly assigned on a 1:1 basis to bevacizumab combined with chemotherapy or only chemotherapy. A best response of complete response or partial response was reported for $21.1 \%$ of patients in the chemotherapy alone arm, and $36.9 \%$ of patients in the bevacizumab + chemotherapy arm. There was one complete response in the bevacizumab + chemotherapy arm (Fig. 2B). Stable disease was reported for $42.1 \%$ in chemotherapy alone arm and $36.8 \%$ in bevacizumab + chemotherapy arm. Progressive disease was $36.8 \%$ in chemotherapy alone arm and $26.3 \%$ in bevacizumab + chemotherapy arm. No difference of toxicities such as febrile neutropenia, hemorrhage, neutropenia, and hypertension was found between the two arms (Table III).

$V E G F$ expression in tumor cell lines. ELISA was performed to determine VEGF expression levels in medium after $72 \mathrm{~h}$ of cell culture. VEGF secretion ranged from 13.39 to $182.7 \mathrm{pg} / 24 \mathrm{~h}$ for the 9 cell lines. The VEGF level in the culture media collected from the dishes of the SPCA1 cells was $13.39 \mathrm{ng} / \mathrm{ml}$, however, in media of A549 cells the VEGF level was over $182.7 \mathrm{ng} / \mathrm{ml}$, about 13 times higher than the level detected in media of SPCA1 cells during the same culture period of time. So we chose SPCA1 and A549 cell lines for further experiments.

Tumor cell proliferation and tumor growth. The tumor cell growth curve showed that the growth of SPCA1 cells and A549 cells had no difference (Fig. 3A); but in vivo tumor growth curves based on measured tumor size, the tumor volumes of SPCA1 cells were significantly smaller than those of A549 cells (Fig. 3B and D).

Vascular phenotypes in tumor tissues. Immunohistochemical analysis showed that blood vessels in A549 tumor tissues consisted of disorganized, tortuous and interconnected vascular plexuses. Quantitative analysis showed that the vessel density in A549 tumor tissue was remarkably increased (Fig. 3C and E).

Tumor cell-derived VEGF promotes cancer metastasis in a mouse model. For the metastasis model, first we labeled the tumor cells with $D i I$, then mice were injected intravenously with $1 \times 10^{5}$ SPCA1 and A549 cells. After 14 days, as shown in Fig. 4, in the lungs, the number of metastatic colonies was higher in A549 group in comparison with SPCA1 group (Fig. 4A). Moreover, the size of the lung lesions were also found to be significantly higher in A549 mice, compared with those in SPCA1 mice (Fig. 4B and C). A two-fold increase in the incidence of lung metastasis in the A549 tumor-bearing mice compared with SPCA1 tumor-bearing mice (Fig. 4D).

Reversal of VEGF induced tumor metastasis by anti-VEGFR1, but not by anti-VEGFR2. In normal lung tissue, VEGFR 1 and VEGFR2 were expressed in the cytoplasm of pneumocytes and endothelial cells (Fig. 5A). These findings provide molecular targets of the tumor-derived VEGF. To further define the receptor signal pathway involved in the development of metastasis, two specific neutralizing anti-mouse VEGFR1 (MF1) and VEGFR2 (DC101) monoclonal antibodies were administered to tumor-bearing mice. After 10 days of treatment, the VEGFinduced tumor metastasis in lungs could be partly prevented by the MF1 antibody. However, anti-VEGFR2 treatment produced no effects on VEGF-induced tumor metastasis (Fig. 5B and C). These findings demonstrate that VEGFR-1-mediated signaling is crucial for tumor metastasis. 
A

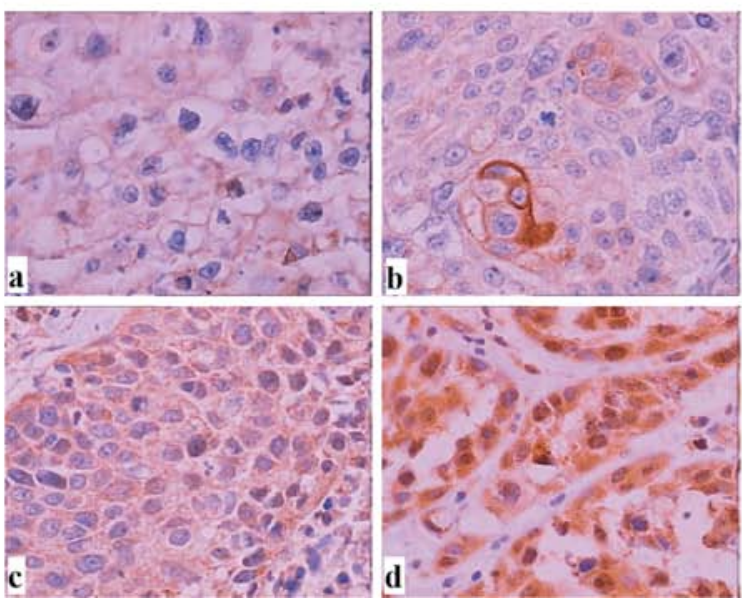

B
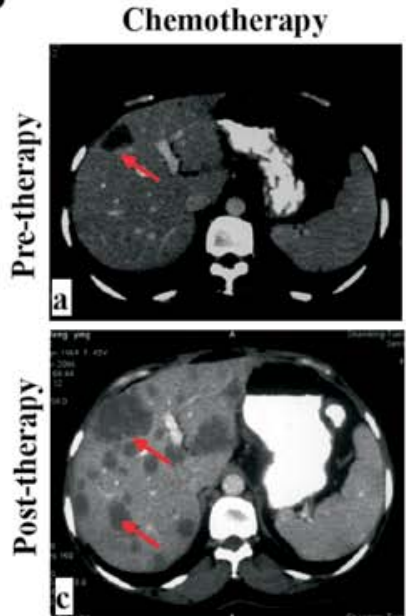

Chemotherapy+beva
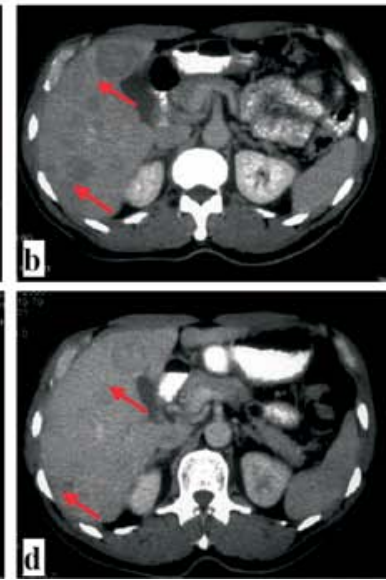

Figure 2. VEGF-A immunohistochemical staining in NSCLC cells and anti-VEGF treatment to cancer patients with liver metastasis. (Aa) no staining of NSCLC cells, (Ab) weak staining of NSCLC cells, (Ac) moderate staining of NSCLC cells, (Ad) strong staining of NSCLC cells; (Ba) One typical patient who received chemotherapy alone, the response was $\mathrm{PD}(\mathrm{Bb}),(\mathrm{Bc})$ one typical patient who received chemotherapy in combination with bevacizumab, the response was PR (Bd).

A

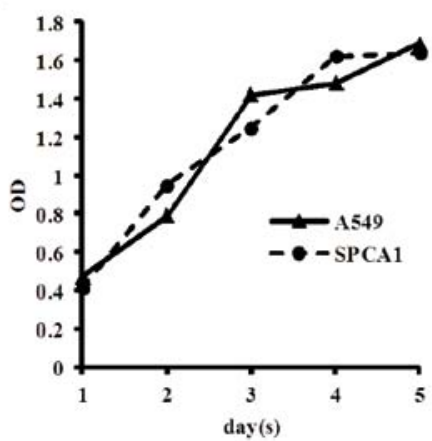

D

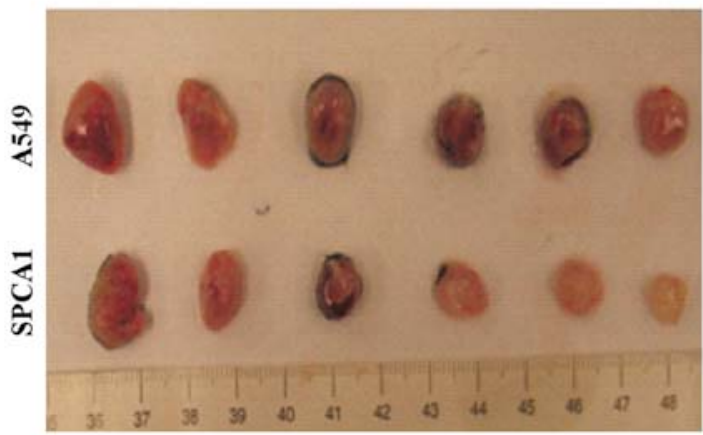

B

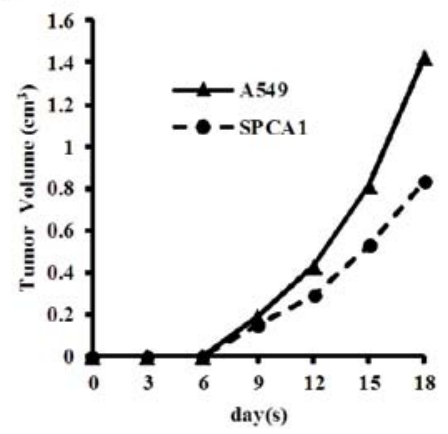

C

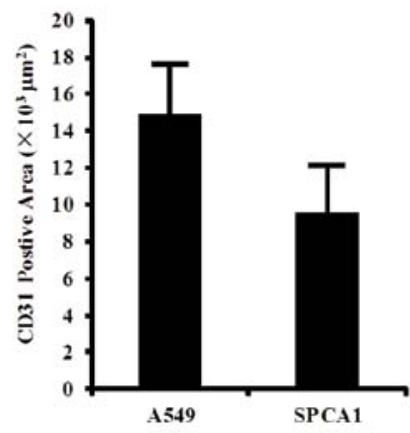

E

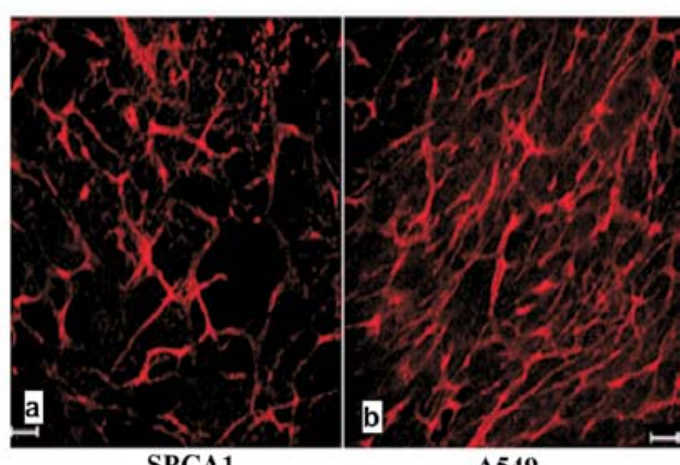

SPCA1

A549

Figure 3. Tumor growth rates and vasculature. (A) The growth curve of SPCA1 cells and A549 cells. (B) Tumor cells were subcutaneously implanted in SCID mice, and tumor growth was measured every 3 days. (C) Quantification of CD31-positive tumor vessels from 5 randomized cryosectioned fields. (D) At day 18 tumors were removed. (E) Tumor tissues were stained with an anti-CD31 antibody, and tumor blood vessels were analyzed by confocal microscopy. Tumor blood vessels are presented in red.

\section{Discussion}

VEGF was originally described as a multifunctional angiogenic regulator that stimulates epithelial cell proliferation, blood vessel formation and endothelial cell survival $(1,5,26)$. Exaggerated levels of VEGF have been detected in serum samples and tumor tissues from patients with cancers, the results of these studies indicated that overexpression of VEGF had a significant correlation with tumor metastasis and worse survival $(27,28)$. In this study, we showed that tumor-derived VEGF facilitated tumor metastasis. In human cancer patients the circulating levels of VEGF in serum were positively correlated with the severity of tumor metastasis, high VEGF expression in tumor tissue was also correlated with tumor 
A

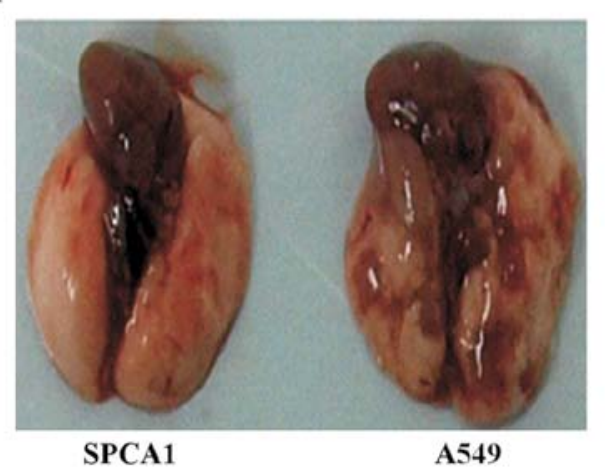

C

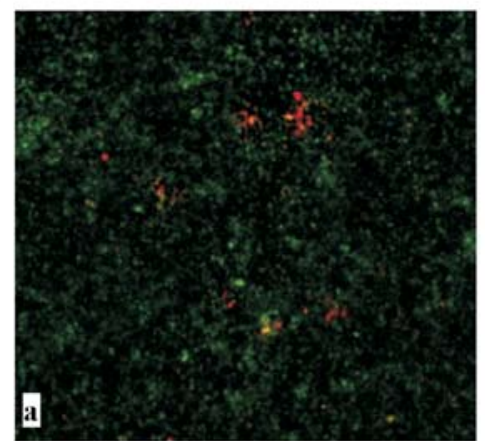

SPCA1

B

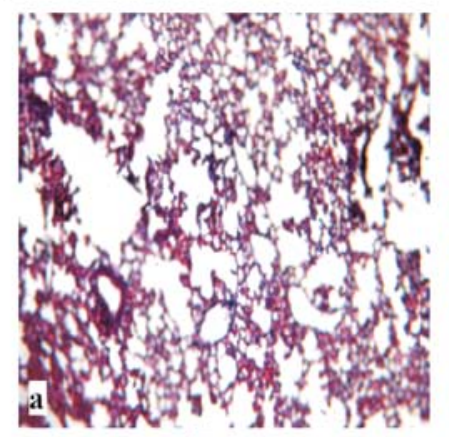

SPCA1

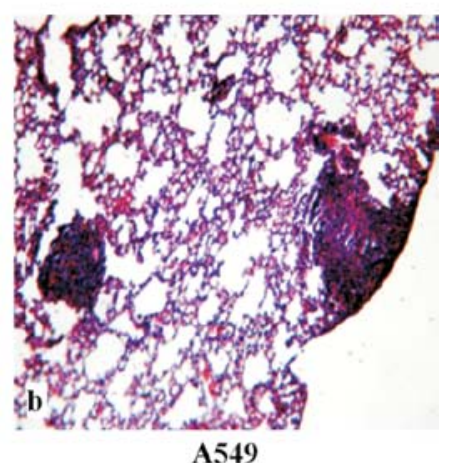

D

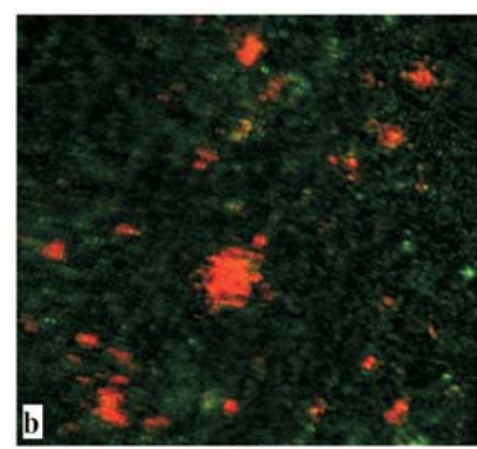

A549

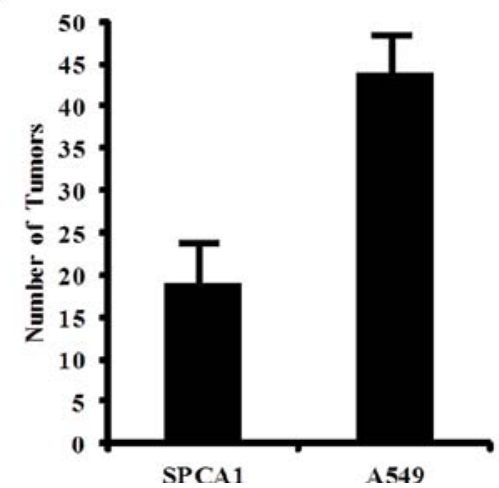

Figure 4. Stimulation of metastasis by expression of VEGF in tumors. (A) visible metastasis in lungs. (B) Lung tissues were stained with HE and metastasis were validated. (C) DiI-postive metastasis in lung tissue. (D) Total number of metastastic foci.

A

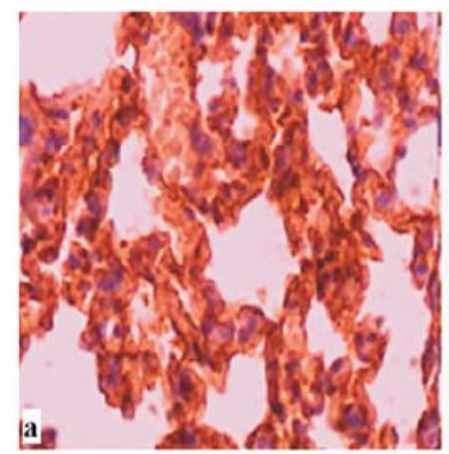

C

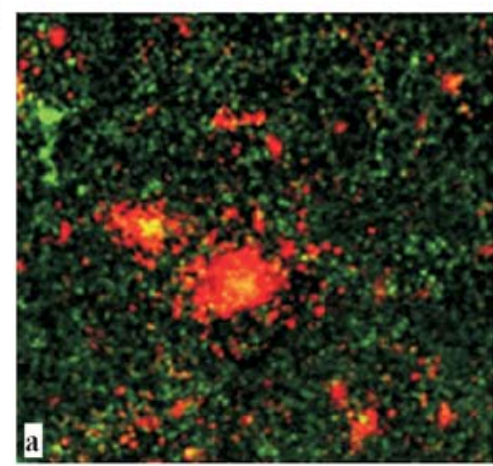

A549

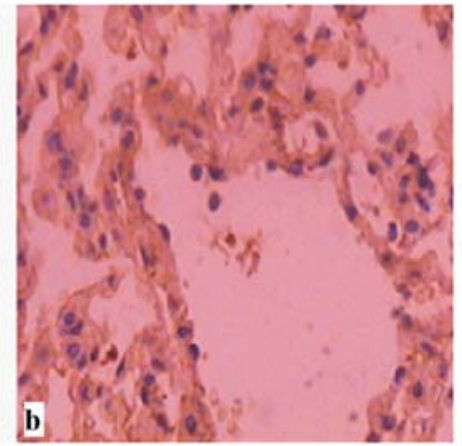

B
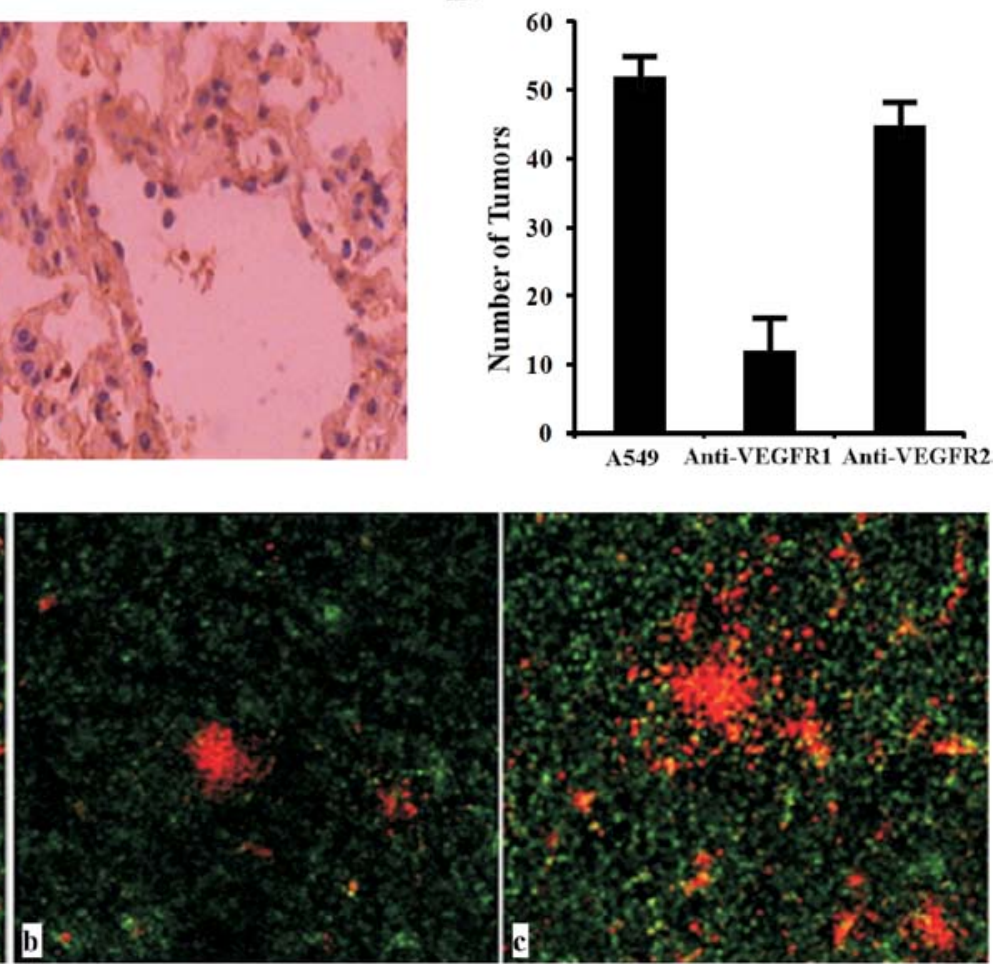

Anti-VEGFR1

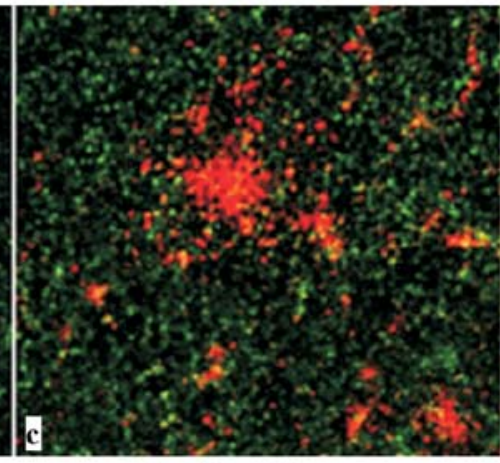

Anti-VEGFR2

Figure 5. Blocking of VEGFRs and tumor metastasis. (Aa) Immunohistochemical staining of normal lung tissue with antibody to VEGFR1; (Ab) Immunohistochemical staining of normal lung tissue with antibody to VEGFR2; (B) Total number of metastastic foci; (C) DiI-postive metastasis in lung tissue. 
metastasis. Bevacizumab is a recombinant humanized, antiVEGF monoclonal antibody that recently received approval by US Food and Drug Administration for use in combination with chemotherapy for patients with unresectable, locally advanced, recurrent or metastastic non-squamous NSCLC. Our study showed that anti-VEGF therapy had significant inhibitive effects on metastatic cancer. It has been shown that the addition of bevacizumab to fluorouracil-based combination chemotherapy results in statistically significant and clinically meaningful improvement in survival among patients with metastatic colorectal cancer (29,30). Our results are consistent with these findings demonstrating that tumor-derived VEGF facilitates tumor metastasis.

To further investigate the correlation of tumor-derived VEGF with metastasis, we used two clonally unrelated mouse lung cancer cell lines to compare the correlation between VEGF expression and metastasis in mouse model. VEGF expressed in the culture media of the SPCA1 cells was $13.39 \mathrm{ng} / \mathrm{ml}$ and in media of A549 cells was over $182.7 \mathrm{ng} / \mathrm{ml}$. In vivo the growth rate of SPCA1 tumors was significantly slower than that of A549 tumors and the number of lung metastasis colonies was considerably higher in A549 group in comparison with SPCA1 group. Yang et al (23) used two human osteosarcoma cell lines with distinct VEGF expressions to investigate the association between the expression of VEGF and the progression of osteosarcoma. The results showed that multiple metastatic lesions were found in the lung tissues of mice in high VEGF group. These studies postulated that the growth, invasion and metastatic potential of tumors were dependent on angiogenesis mediated via the potent proangiogenic factor VEGF.

VEGF mediates its responses primarily by activating VEGFR-1 and VEGFR-2. VEGFR-1 signaling plays an important role in angiogenesis under pathological conditions $(1,2,31)$. VEGFR-2 is the major mediator of the mitogenic angiogenic and permeability enhancing the effect of VEGF. VEGF impairs the endothelial barrier by disrupting a VE-cadherin/ $\beta$-catenin complex via the activation of Src and facilitates tumor cell extravasation and metastasis (32). VEGF also induces the disruption of hepatocellular tight junctions, which may facilitate tumor cell invasion and metastasis (33). In our study, the results showed high levels of both VEGFR-1 and VEGFR-2 in the lung tissues. These findings provide molecular targets of the tumor VEGF-induced metastasis. Two specific neutralizing anti-mouse VEGFR-1 (MF1) and VEGFR-2 (DC101) monoclonal antibodies were administered to the tumor-bearing mice, the VEGF-induced tumor metastasis in lung could be partly prevented by anti-VEGFR1 antibody, and however, anti-VEGFR-2 treatment produced no effects on VEGF-induced tumor metastasis. These findings demonstrate that VEGFR-1-mediated signal is crucial for tumor metastasis.

Although there are many studies in the field of VEGF and tumor angiogenesis, but there are only a few reports concerning the correlation between VEGF-VEGFR pathway and tumor metastasis. Lin et al (34) have reported that VEGF stimulated lymphangiogenesis through the VEGF-C/-D/ VEGFR-3 independent pathway. Thus, our work together with this report provides novel insights into the understanding of the role of VEGF in facilitating primary tumor growth and tumor metastasis. Blockage of VEGF or VEGFR1 may provide novel approaches for prevention and treatment of tumor metastasis.

\section{Acknowledgements}

This study was supported by grants from the Shandong Province Science and Technology Project, no. 2007GG20002007; no. BS2009SW050; 2009HZ063.

\section{References}

1. Ferrara N, Gerber HP and LeCouter J: The biology of VEGF and its receptors. Nat Med 9: 669-676, 2003.

2. Ferrara N: The role of VEGF in the regulation of physiological and pathological angiogenesis. EXS 94: 209-231, 2005.

3. Sato Y, Kanno S, Oda N, Abe M, Ito M, Shitara K and Shibuya M: Properties of two VEGF receptors, Flt-1 and KDR, in signal transduction. Ann NY Acda Sci 902: 201-205, 2000.

4. Lohela M, Bry M, Tammela T and Alitalo K: VEGFs and receptors involved in angiogenesis versus lymphangiogenesis. Curr Opin Cell Biol 21: 154-165, 2009.

5. Lee S, Chen TT, Barber CL, Jordan MC, Murdock J, Desai S, Ferrara N, Nagy A, Roos KP and Iruela-Arispe ML: Autocrine VEGF signaling is required for vascular homeostasis. Cell 130: 691-703, 2007.

6. Fukasawa M and Korc M: Vascular endothelial growth factortrap suppresses tumorigenicity of multiple pancreatic cancer cell lines. Clin Cancer Res 10: 3327-3332, 2004.

7. Carmeliet P and Jain RK: Angiogenesis in cancer and other diseases. Nature 407: 249-257, 2000.

8. Ferrara N: Role of myeloid cells in vascular endothelial growth factor independent tumor angiogenesis. Curr Opin Hematol 17: 219-224, 2010.

9. Geiger TR and Peeper DS: Metastasis mechanisms. Biochim Biophys Acta 1796: 293-308, 2009.

10. Jechlinger M, Sommer A, Moriggl R, Seither P, Kraut N, Capodiecci P, Donovan M, Cordon-Cardo C, Beug H and Grünert S: Autocrine PDGFR signaling promotes mammary cancer metastasis. J Clin Invest 116: 1561-1570, 2006.

11. Fidler IJ: The pathogenesis of cancer metastasis: the 'seed and soil' hypothesis revisited. Nat Rev Cancer 3: 453-458, 2003.

12. Yang $\mathbf{J}$ and Weinberg RA: Epithelial-mesenchymal transition: at the crossroads of development and tumor metastasis. Dev Cell 14: 818-829, 2008.

13. Pantel K, Brakenhoff RH and Brandt B: Detection, clinical relevance and specific biological properties of disseminating tumour cells. Nat Rev Cancer 8: 329-340, 2008.

14. Caceres W and Gonzalez S: Angiogenesis and cancer: recent advances. PR Health Sci J 22: 149-151, 2003.

15. Carmeliet P: Angiogenesis in life, disease and medicine. Nature 438: 932-936, 2005.

16. Maharaj AS, Saint-Geniez M, Maldonado AE and D'Amore PA: Vascular endothelial growth factor localization in the adult. Am J Pathol 168: 639-648, 2006.

17. Bjorndahl MA, Cao R, Burton JS, Brakenhielm E, Religa P, Galter D, Wu L and Cao Y: Vascular endothelial growth factor-A promotes peritumoral lymphangiogenesis and lymphatic metastasis. Cancer Res 65: 9261-9268, 2005.

18. Weis S, Cui J, Barnes L and Cheresh D: Endothelial barrier disruption by VEGF-mediated Src activity potentiates tumor cell extravasation and metastasis. J Cell Biol 167: 223-229, 2004.

19. Lee YH, Tokunaga T, Oshika Y, Suto R, Yanagisawa K, Tomisawa M, Fukuda H, Nakano H, Abe S, Tateishi A, Kijima H, Yamazaki H, Tamaoki N, Ueyama Y and Nakamura M. Cell retained isoforms of vascular endothelial growth factor (VEGF) are correlated with poor prognosis in osteosarcoma. Eur J Cancer 35: 1089-1093, 1999.

20. Hirakawa S, Kodama S, Kunstfeld R, Kajiya K, Brown LF and Detmar M: VEGF-A induces tumor and sentinel lymph node lymphangiogenesis and promotes lymphatic metastasis. J Exp Med 201: 1089-1099, 2005.

21. Saito DM, Franco MR, Parra ER, Ab'saber AM, Farhat C, Eher E, Soufen MA, Rodrigues R, Capelozzi VL: Cell cycle regulator in primary lung adenocarcinoma and its haematogenous metastases. Histopathology 50: 525-527, 2007.

22. Alitalo K, Tammela T and Petrova TV: Lymphangiogenesis in development and human disease. Nature 438: 946-953, 2005. 
23. Yang SY, Yu H, Krygier JE, Wooley PH and Mott MP: High VEGF with rapid growth and early metastasis in mouse osteosarcoma model. Sarcoma 2007: 95628, 2007.

24. Sullivan R and Graham CH: Hypoxia-driven selection of the metastatic phenotype. Cancer Metastasis Rev 26: 319-331, 2007.

25. Ferrara $\mathrm{N}$ and Kerbel RS: Angiogenesis as a therapeutic target. Nature 438: 967-974, 2005.

26. Takahashi $\mathrm{H}$ and Shibuya $\mathrm{M}$ : The vascular endothelial growth factor (VEGF)/VEGF receptor system and its role under physiological and pathological conditions. Clinical Science 109: 227-241, 2005.

27. Decaussin M, Sartelet H, Robert C, Moro D, Claraz C, Brambilla $\mathrm{C}$ and Brambilla E: Expression of vascular endothelial growth factor (VEGF) and its two receptors (VEGF-R1-Flt1 and VEGF- R2-Flk1/KDR) in non-small cell lung carcinomas (NSCLCs): correlation with angiogenesis and survival. J Pathol 188: 369-377, 1999.

28. Kaya M, Wada T, Akatsuka T, Kawaguchi S, Nagoya S, Shindoh M, Higashino F, Mezawa F, Okada F and Ishii S: Vascular endothelial growth factor expression in untreated osteosarcoma is predictive of pulmonary metastasis and poor prognosis. Clin Cancer Res 6 : $572-577,2000$

29. Sharieff W: Bevacizumab in colorectal cancer. N Engl J Med 351: 1690-1691, 2004.
30. Hirsh V: Systemic therapies in metastatic non-small cell lung cancer with emphasis on targeted therapies: the rational approach. Curr Oncol 17: 13-23, 2010

31. Hiratsuka S, Nakamura K, Iwai S, Murakami M, Itoh T, Kijima H, Shipley JM, Senior RM and Shibuya M: MMP9 induction by vascular endothelial growth factor receptor-1 is involved in lungspecific metastasis. Cancer Cell 2: 289-300, 2002.

32. Hiratsuka S, Nakao K, Nakamura K, Katsuki M, Maru Y and Shibuya M: Membrane fixation of vascular endothelial growth factor receptor 1 ligand-binding domain is important for vasculogenesis and angiogenesis in mice. Mol Cell Biol 25: 346-354, 2005.

33. Zhang D, Li B, Shi J, Zhao L, Zhang X, Wang C, Hou S, Qian W, Kou G, Wang H and Guo Y: Suppression of tumor growth and metastasis by simultaneously blocking vascular endothelial growth factor (VEGF)-A and VEGF-C with a receptor- immunoglobulin fusion protein. Cancer Res 70: 2495-2503, 2010.

34. Lin J, Lalani AS, Harding TC, Gonzalez M, Wu WW, Luan B, Tu GH, Koprivnikar K, VanRoey MJ, He Y, Alitalo K and Jooss K: Inhibition of lymphogenous metastasis using adeno-associated virus-mediated gene transfer of a soluble VEGFR-3 decoy receptor. Cancer Res 65: 6901-6909, 2005. 